\title{
Food and Nutrition Literacy (FNLIT) is Associated to Healthy Eating Behaviors in Children
}

\author{
Azam Doustmohammadian ${ }^{1}$, Nasrin Omidvar*2 ${ }^{2}$, Nastaran Keshavarz Mohammadi ${ }^{3}$, Hassan Eini-Zinab ${ }^{2}$, Maryam Amini ${ }^{4}$, Morteza \\ Abdollahi $^{4}$, Saeed Esfandiari ${ }^{2}$ Zeinab Amirhamidi ${ }^{2}$ \\ 1- Gastrointestinal and Liver Diseases Research Center, Iran University of Medical Sciences, Tehran, Iran \\ 2- Department of Community Nutrition, National Nutrition and Food Technology Research Institute (WHO Collaborating Center); and Faculty of \\ Nutrition Sciences and Food Technology, Shahid Beheshti University of Medical Sciences, Tehran, Iran \\ 3- School of Public Health, Shahid Beheshti University of Medical Sciences, Tehran, Iran \\ 4- Department of Nutrition Research, National Nutrition and Food Technology Research Institute (WHO Collaborating Center); and Faculty of Nutrition \\ Sciences and Food Technology, Shahid Beheshti University of Medical Sciences, Tehran, Iran
}

\section{A B S T R A C T}

Background and Objectives: The objective of the current study was to investigate associations between food and nutrition literacy (FNLIT) and eating behaviors of elementary school children in Tehran, Iran.

Materials and Methods: In this cross-sectional study, 803 students aged 10-12 years were participated from 44 primary schools in Tehran, Iran. A valid, reliable self-administered questionnaire was used to investigate participants' food and nutrition literacy. A research-made questionnaire was used to assess eating behaviors of the students. Furthermore, the multinomial adjusted odds ratios of food and nutrition literacy for eating behaviors were analyzed.

Results: Food and nutrition literacy cognitive domain included understanding food and nutrition information and nutritional health knowledge. Food and nutrition literacy skill domain included functional, interactive, critical food and nutrition literacy, food choice and food label literacy. High levels of food and nutrition literacy scores in the cognitive domain were negatively associated to irregular breakfast intakes compared to everyday eating breakfast (1-2 times a week, $\mathrm{OR}=0.32, \mathrm{CI}=0.17-0.60$ ), irregular lunch intakes compared to everyday eating lunch (never/1-2 times a week, $\mathrm{OR}=$ $0.40, \mathrm{CI}=0.17-0.93$; 3-6 times a week, $\mathrm{OR}=0.45, \mathrm{CI}=0.25-0.80)$ and irregular dinner intakes compared to everyday eating dinner (never/1-2 times a week, $\mathrm{OR}=0.32, \mathrm{CI}=0.17-0.68$ ). High food and nutrition literacy scores in the cognitive domain were attributed to never eating sausage/hamburger $(\mathrm{OR}=2.20, \mathrm{CI}=1.01-4.83)$ and eating salty snacks 3-4 times a week $(\mathrm{OR}=2.58, \mathrm{CI}=1.09-6.13)$. The FNLIT scores in the skill domain were negatively associated to irregular breakfast intakes compared to everyday eating breakfast $(3-6$ times a week, $\mathrm{OR}=0.33, \mathrm{CI}=0.13-0.78)$. Food and nutrition literacy scores in the skill domain were positively associated to never eating sweet snacks $(\mathrm{OR}=4.19$, $\mathrm{CI}=1.39-12.62)$.

Conclusions: The current manuscript highlights the necessity of continuous improvements in health education curriculum of schools in Iran, particularly highlighting the importance of greater attention needs to practical and skillbased lessons rather than theoretical lessens. Further studies with long-term follow-up plans are needed to understand associations between food and nutrition literacy and eating behaviors more comprehensively.

Keywords: Food and Nutrition literacy, Eating behaviors, School-age children, Iran

\section{Introduction}

Urbanization and development have resulted in major lifestyle changes, including dietary behaviors and physical activities in developed and developing countries. Poor dietary habits have been associated to diet-related chronic diseases (DRCD), including obesity and diabetes $(1,2)$. As a country experiencing nutrition transition, Iran witnesses

major behaver changes in adults and children. High-risk nutritional behaviors such as meal skipping, unhealthy dietary habits and low physical activities increase in the country (3-5). Childhood provides opportunities for health promotion to lead the adoption of healthy behaviors, preventing health problems in adulthood (6). Health 
literacy is reported as one of the most important personal skills to enable individuals to control health determinants (7). Evidence suggest needs of emphasizing specific areas of health literacy, including food literacy/nutrition literacy, due to the wide scopes of health issues. Food and nutrition literacies have recently emerged concepts, which address knowledge and skills of food and nutrition $(8,9)$. Overall, transition from knowledge to practice is described as a vital component of either food or nutrition literacy (10).

Current studies have shown that food and nutrition literacy is one of the key factors in forming eating behaviors of children and adolescents (11-13). Food skills such as cutting fruits and vegetables, following recipes, measuring ingredients and preparing foods are shown to be associated to increased consumption of fruits and vegetables in adolescents (14). Food and nutrition literacy such as health literacy can be conceptualized as an asset (15) or enabler to healthy eating (9). Clarifying magnitude and nature of the relationships between food and nutrition literacy and children dietary behaviors is important to develop effective strategies for intervention in this group. In addition, it may help predict effects of interventions that focus on improving food literacy to improve children dietary habits. Therefore, the aim of this study was to assess relationships between food and nutrition literacy and dietary habits in primary school children in the metropolitan city of Tehran, Iran.

\section{Materials and Methods}

\section{Study design and participants}

This study was a population-based cross-sectional survey using multistage random cluster sampling design. Study sample included 803 primary school students of 419 boys and 384 girls aged 10-12 years (power study, 88\%; response rate, $89.2 \%$ ) from various socioeconomic districts of Tehran, Iran. Students and their parents were provided with written informed consents before beginning of the survey. Data collection was carried out at schools by trained research assistants. The STROBE study on food and nutrition literacy (FNLIT) and dietary habits in children is outlined in Fig. 1.

\section{Measurements}

\section{Food and nutrition literacy assessment}

A valid self-administered questionnaire was used for the assessment of FNLIT. The process of developing questionnaire and assessing its interface, content and construct validity has previously been described (16). The FNLIT questionnaire included 46 items in the cognitive and skill domains. The cognitive domain included two subscales of understanding food and nutrition information (ten items) and nutritional health knowledge (five items). The skill domain included four subscales of functional food and nutrition literacy (ten items), interactive food and nutrition literacy (seven items), food choice (six items) and critical food and nutrition literacy (four items). Food label literacy was assessed by four true-false items. The FNLIT status was categorized into three levels of low $(\leq 51)$, medium (> 51 to $<74)$ and high $(\geq 74)$ categories $(17)$, where the FNLIT score ranged 25.8-96.8.

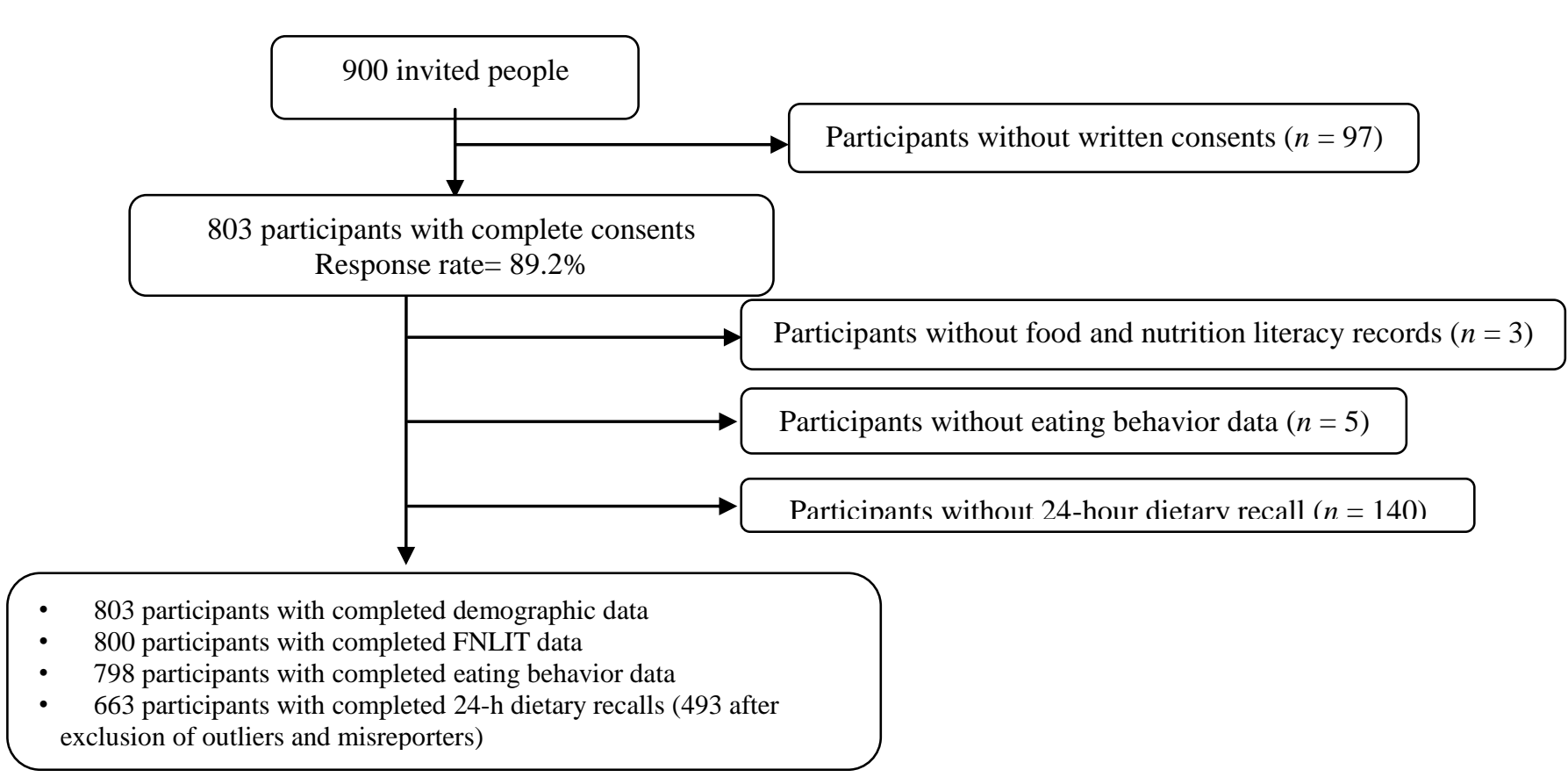

Figure 1. The STROBE study flow chart 


\section{Study covariates}

In this study, several baseline covariates were addressed based on the available evidence $(8,23-25)$. Physical activity was assessed using a locally validated version of the child and adolescent international physical activity questionnaire and interviews by trained research assistants $(26,27)$. Household food security status was assessed using locally validated 18 -item USDA household food security survey module and face-to-face interviews with mothers $(28,29)$. Calorie intake was assessed using three 24-h recalls (two week days and one holiday) by trained nutritionists. Demographic and socioeconomic characteristics were collected using questionnaires and interviews with students and then verified by their mothers and/or caregivers.

\section{Statistical analysis}

Normality of distribution was assessed using Kolmogorov-Smirnov test. Data were presented as frequencies and percentages for categorical variables. Chisquare test was used for the analysis of general characteristics of the participants, eating behaviors categories and FNLIT categories in the cognitive and skill domains. Multinomial logistic regression adjusted for covariates was used to estimate associations between the FNLIT and eating behaviors. Two-tailed tests were used and $p$-values less than 0.05 were considered as statistically significant. All statistical analyses were carried out using SPSS Software v.21.0 (SPSS, Chicago, Illinois, USA).

\section{Results}

\section{Characteristics of the study participants}

The participants' general characteristics are presented in Tables 1 and 2 based on the FNLIT scores in congnitive and skill domains. Of the total students, $68.8 \%$ included high-level cognitive domains. At least one out of four students $(25 \%)$ included low FNLIT values in the skill domain and a very few students included low scores in the cognitive domain $(2.6 \%)$. The cognitive domain scores of FNLIT were significantly associated to father age, family size, mother education and student weight status. For example, $10 \%$ of students with a small family size $(<4$ people) included low cognitive domain scores, compared to those with a family size of $4(50 \%)$ and larger (40\%). No significant relationships were seen between the demographic and socioeconomic characteristics and the FNLIT skill domain scores. Due to the low prevalence of FI with moderate and severe hunger, these values were grouped as one. No significant relationships were observed between the household food security status and the FNLIT skill and cognitive domain scores.

\section{Association between FNLIT and eating behaviors}

Children eating behaviors are summarized in Table 3. Irregular breakfast intakes were reported in nearly $34 \%$ of the students. Based on the results from Tables 4 and 5, high FNLIT scores in cognitive and skill domains were positively associated to eating breakfast, compared to irregular breakfast intake. High FNLIT scores in cognitive domain were positively associated to eating lunch every day compared to less than seven days a week, and to regular dinner intake compared to having dinner less than seven days a week. High FNLIT scores in knowledge domain increased possibilities of eating salty snacks 3-4 times a week, while high FNLIT scores in the skill domain were associated to never eating sweet snacks.

Table 1. General characteristics of the participants based on the food and nutrition literacy scores in congnitive and skill domains

\begin{tabular}{|c|c|c|c|c|c|c|c|c|c|}
\hline & \multirow[b]{3}{*}{ Total } & \multicolumn{4}{|c|}{ Food and Nutrition Literacy } & \multicolumn{4}{|c|}{ Food and Nutrition Literacy } \\
\hline & & \multicolumn{4}{|c|}{ Cognitive domain } & \multicolumn{4}{|c|}{ Skill domain } \\
\hline & & Low & Medium & high & P value* & low & Medium & high & $\mathrm{P}$ value* \\
\hline & $\mathrm{N}(\%)$ & $\mathrm{N}(\%)$ & $\mathrm{N}(\%)$ & $\mathrm{N}(\%)$ & & $\mathrm{N}(\%)$ & $\mathrm{N}(\%)$ & $\mathrm{N}(\%)$ & \\
\hline Overall & 800 & $21(2.6)$ & $229(28.6)$ & $550(68.8)$ & & $200(25.0)$ & $479(59.9)$ & $121(15.1)$ & \\
\hline Sex & 800 & & & & $0.05^{*}$ & & & & 0.96 \\
\hline Female & $381(47.6)$ & $6(28.6)$ & $100(43.7)$ & $275(50.0)$ & & $94(47.0)$ & $230(48.0)$ & $57(47.1)$ & \\
\hline Male & $419(52.4)$ & $15(71.4)$ & $129(56.3)$ & $275(50.0)$ & & $106(53.0)$ & $249(52.0)$ & $64(52.9)$ & \\
\hline Grade & 800 & & & & 0.31 & & & & 0.53 \\
\hline Fifth & $413(51.6)$ & $14(66.7)$ & $121(52.8)$ & $278(50.5)$ & & $99(49.5)$ & $255(53.2)$ & $59(48.8)$ & \\
\hline Sixth & $387(48.4)$ & $7(33.3)$ & $108(47.2)$ & $272(49.5)$ & & $101(50.0)$ & $224(46.8)$ & $62(51.2)$ & \\
\hline
\end{tabular}


Azam Doustmohammadian, et al: Food and Nutrition Literacy (FNLIT) and eating behavior

\begin{tabular}{|c|c|c|c|c|c|c|c|c|c|}
\hline \multirow{4}{*}{ Table 1 (continued). } & \multirow[b]{3}{*}{ Total } & \multicolumn{4}{|c|}{ Food and Nutrition Literacy } & \multicolumn{4}{|c|}{ Food and Nutrition Literacy } \\
\hline & & \multicolumn{4}{|c|}{ Cognitive domain } & \multicolumn{4}{|c|}{ Skill domain } \\
\hline & & Low & Medium & high & P value* & low & Medium & high & P value* \\
\hline & $\mathrm{N}(\%)$ & $\mathrm{N}(\%)$ & $\mathrm{N}(\%)$ & $\mathrm{N}(\%)$ & & $\mathrm{N}(\%)$ & $\mathrm{N}(\%)$ & $\mathrm{N}(\%)$ & \\
\hline Birth order & 798 & & & & 0.47 & & & & 0.21 \\
\hline 1 & $437(54.8)$ & $12(60.0)$ & $118(51.5)$ & $307(55.9)$ & & $107(54.0)$ & $255(53.2)$ & $75(62.0)$ & \\
\hline$>1$ & $361(45.2)$ & $8(40.0)$ & $111(48.5)$ & $242(44.1)$ & & $91(46.0)$ & $224(46.8)$ & $46(38.0)$ & \\
\hline Father age tertile (year) & 790 & & & & $0.04 *$ & & & & 0.63 \\
\hline $30-40$ & $300(38)$ & $14(66.7)$ & $90(40.2)$ & $196(36.0)$ & & $75(38.5)$ & $179(37.6)$ & $46(38.7)$ & \\
\hline $41-45$ & $265(33.5)$ & $3(14.3)$ & $68(30.4)$ & 194(35.6) & & $63(32.3)$ & $168(35.3)$ & $34(28.6)$ & \\
\hline$\geq 46$ & $225(28.5)$ & $4(19.0)$ & $66(29.5)$ & $155(28.4)$ & & $57(29.2)$ & $129(27.1)$ & $39(32.8)$ & \\
\hline Mother age tertile (year) & 794 & & & & 0.50 & & & & 0.12 \\
\hline $23-35$ & $288(36.3)$ & $10(47.6)$ & $89(39.2)$ & $189(34.6)$ & & $79(40.1)$ & $158(33.2)$ & $51(42.1)$ & \\
\hline $36-40$ & $303(38.2)$ & $8(38.1)$ & $82(36.1)$ & $213(39.0)$ & & $76(38.6)$ & 190(39.9) & $37(30.6)$ & \\
\hline$\geq 41$ & $203(25.6)$ & $3(14.3)$ & $56(24.7)$ & $144(26.4)$ & & $42(21.3)$ & $128(26.9)$ & $33(27.3)$ & \\
\hline Ethnicity & 797 & & & & 0.49 & & & & 0.95 \\
\hline Fars & $441(55.3)$ & $8(40.0)$ & $128(55.9)$ & $305(55.7)$ & & $108(54.3)$ & $263(55.1)$ & $70(57.9)$ & \\
\hline Azeri & $228(28.3)$ & $9(45.0)$ & 64(27.9) & $155(28.3)$ & & $57(28.6)$ & 141(29.6) & $30(24.8)$ & \\
\hline Fars-Azeri & $56(7)$ & $0(0)$ & $18(7.9)$ & $38(6.9)$ & & $14(7.0)$ & $32(6.7)$ & $10(8.3)$ & \\
\hline Other & $72(9)$ & $3(15.0)$ & $19(8.3)$ & $50(9.1)$ & & $20(10.1)$ & $41(8.6)$ & $11(9.1)$ & \\
\hline School status & 800 & & & & 0.48 & & & & 0.41 \\
\hline Public & $725(90.6)$ & $19(90.5)$ & 212(92.6) & $494(89.8)$ & & 184(92.0) & $435(90.8)$ & 106(87.6) & \\
\hline Private & $75(9.4)$ & $2(9.5)$ & $17(7.4)$ & $56(10.2)$ & & $16(8.0)$ & $44(9.2)$ & $15(12.4)$ & \\
\hline Family size & 797 & & & & $0.02 *$ & & & & 0.28 \\
\hline$<4$ & $160(20.1)$ & $2(10.0)$ & $44(19.2)$ & $114(20.8)$ & & $42(21.3)$ & $90(18.8)$ & $28(23.1)$ & \\
\hline 4 & $465(58.3)$ & $10(50.0)$ & $123(53.7)$ & $332(60.6)$ & & $106(53.8)$ & $285(59.5)$ & $74(61.2)$ & \\
\hline$>4$ & $172(21.6)$ & $8(40.0)$ & $62(27.1)$ & $102(18.6)$ & & 49(24.9) & $104(21.7)$ & $19(15.7)$ & \\
\hline Father education & 789 & & & & 0.28 & & & & 0.63 \\
\hline illiterate or $\leq 5$ years & $85(10.8)$ & $3(14.3)$ & $26(11.6)$ & $56(10.3)$ & & $23(11.8)$ & $54(11.4)$ & $8(6.7)$ & \\
\hline 6-9 years or diploma & $395(50.1)$ & $11(52.4)$ & $123(54.9)$ & $261(48.0)$ & & $98(50.3)$ & $236(49.7)$ & $61(51.3)$ & \\
\hline $\begin{array}{l}\text { associate's degree or } \\
\text { higher }\end{array}$ & $309(39.2)$ & $7(33.3)$ & $75(33.5)$ & $227(41.7)$ & & $74(37.9)$ & $185(38.9)$ & $50(42.0)$ & \\
\hline Mother education & 794 & & & & $0.004 *$ & & & & 0.07 \\
\hline illiterate or $\leq 5$ years & $86(10.8)$ & $1(4.8)$ & $21(9.3)$ & $64(11.7)$ & & 21(10.7) & $52(10.9)$ & $13(10.7)$ & \\
\hline 6-9 years or diploma & $461(58.1)$ & $15(71.4)$ & $154(67.8)$ & $292(53.5)$ & & $129(65.5)$ & $270(56.7)$ & $62(51.2)$ & \\
\hline $\begin{array}{l}\text { associate's degree or } \\
\text { higher }\end{array}$ & $247(31.1)$ & $5(23.8)$ & $52(22.9)$ & $190(34.8)$ & & $47(23.9)$ & $154(32.4)$ & $46(38.0)$ & \\
\hline Father job position & 778 & & & & 0.48 & & & & 0.67 \\
\hline Worker & 106(13.6) & $2(9.5)$ & $35(16.1)$ & $69(12.8)$ & & $29(15.3)$ & $64(13.6)$ & 13(11.0) & \\
\hline employee & $327(42)$ & 13(61.9) & $84(38.5)$ & $230(42.7)$ & & $82(43.2)$ & 191(40.6) & $54(45.8)$ & \\
\hline high rank employee & 139(17.9) & $1(4.8)$ & $37(17.0)$ & 101(18.7) & & $28(14.7)$ & $93(19.8)$ & $18(15.3)$ & \\
\hline Retired & $20(2.6)$ & $1(4.8)$ & $6(2.8)$ & $13(2.4)$ & & $5(2.6)$ & $10(2.1)$ & $5(4.2)$ & \\
\hline self-manager & $186(23.9)$ & $4(19.0)$ & $56(25.7)$ & $126(23.4)$ & & $46(24.2)$ & $112(23.8)$ & $28(23.7)$ & \\
\hline Mother employment & 794 & & & & 0.27 & & & & 0.88 \\
\hline Working & $630(79.3)$ & $18(85.7)$ & 187(82.4) & $425(77.8)$ & & $154(78.2)$ & $379(79.6)$ & $97(80.2)$ & \\
\hline housewife & $164(20.7)$ & $3(14.3)$ & 40(17.6) & $121(22.2)$ & & $43(21.8)$ & $97(20.4)$ & $24(19.8)$ & \\
\hline House ownership status & 799 & & & & 0.22 & & & & 0.44 \\
\hline Owner & $427(53.4)$ & $10(47.6)$ & $127(55.5)$ & $290(53.4)$ & & $101(50.5)$ & $256(53.6)$ & $70(57.9)$ & \\
\hline Tenant & $262(32.8)$ & $8(38.1)$ & $80(34.9)$ & $174(31.7)$ & & $77(38.5)$ & $152(31.8)$ & $33(27.3)$ & \\
\hline mortgage & $35(4.4)$ & $0(0)$ & $10(4.4)$ & $25(4.6)$ & & $8(4.0)$ & $21(4.4)$ & $6(5.0)$ & \\
\hline Other & $75(9.4)$ & $3(14.3)$ & $12(5.2)$ & $60(10.9)$ & & $14(7.0)$ & $49(10.3)$ & $12(9.9)$ & \\
\hline
\end{tabular}


Table 2. Food security, physical activity, weight status and energy intake characteristics of the participants based on the food and nutrition literacy scores in congnitive and skill domains

\begin{tabular}{|c|c|c|c|c|c|c|c|c|c|}
\hline & \multirow[b]{3}{*}{ Total } & \multicolumn{4}{|c|}{ Food and Nutrition Literacy } & \multicolumn{4}{|c|}{ Food and Nutrition Literacy } \\
\hline & & \multicolumn{4}{|c|}{ Cognitive domain } & \multicolumn{4}{|c|}{ Skill domain } \\
\hline & & Low & Medium & high & $\mathrm{P}$ value* & low & Medium & high & $\mathrm{P}$ value* \\
\hline & $\mathrm{N}(\%)$ & $\mathrm{N}(\%)$ & $\mathrm{N}(\%)$ & $\mathrm{N}(\%)$ & & $\mathrm{N}(\%)$ & $\mathrm{N}(\%)$ & $\mathrm{N}(\%)$ & \\
\hline Overall & 800 & $21(2.6)$ & $229(28.6)$ & $550(68.8)$ & & $200(25.0)$ & $479(59.9)$ & $121(15.1)$ & \\
\hline $\mathbf{H H}^{1}$ food security status & 639 & & & & 0.52 & & & & 0.05 \\
\hline $\mathrm{FS}^{2}$ & $481(75.3)$ & $10(66.7)$ & $136(73.9)$ & $335(76.1)$ & & $117(72.7)$ & $285(73.8)$ & $79(85.9)$ & \\
\hline $\mathrm{FI}^{3}$ without hunger & $111(17.4)$ & $4(26.7)$ & $30(16.3)$ & $77(17.5)$ & & $32(19.9)$ & $67(17.4)$ & $12(13.0)$ & \\
\hline FI with hunger & $47(7.4)$ & $1(6.7)$ & $18(9.8)$ & $28(6.4)$ & & $12(7.5)$ & $34(8.8)$ & $1(1.1)$ & \\
\hline Physical activity tertile MET.h/day) & 787 & & & & 0.47 & & & & 0.28 \\
\hline Mean T1: 33 & $260(33.0)$ & $6(28.6)$ & $75(33.5)$ & $179(33.0)$ & & $74(37.4)$ & $155(32.9)$ & $31(26.3)$ & \\
\hline Mean T2: 38.37 & $262(33.3)$ & $6(28.6)$ & $66(29.5)$ & $190(35.1)$ & & $57(28.8)$ & $160(34.0)$ & $45(38.1)$ & \\
\hline Mean T3: 47.71 & $265(33.7)$ & $9(42.9)$ & $83(37.1)$ & $173(31.9)$ & & $67(33.8)$ & $156(33.1)$ & $42(35.6)$ & \\
\hline Weight statue (BMI Z scores) & 800 & & & & $0.02 *$ & & & & 0.85 \\
\hline Thin & $15(1.9)$ & $2(9.5)$ & $6(2.6)$ & $7(1.3)$ & & $5(2.5)$ & $9(1.9)$ & $1(0.8)$ & \\
\hline Normal & $381(47.6)$ & $12(57.1)$ & $118(51.5)$ & $251(45.6)$ & & $100(50.0)$ & $222(46.3)$ & $599(48.8)$ & \\
\hline Overweight & $213(26.6)$ & $2(9.5)$ & $58(25.3)$ & $153(27.8)$ & & $47(23.5)$ & $133(27.8)$ & $33(27.3)$ & \\
\hline Obese & 191(23.9) & $5(23.8)$ & $47(20.5)$ & $139(25.3)$ & & $489(24.0)$ & $115(24.0)$ & $28(23.1)$ & \\
\hline Energy intake tertile (kcal/day) & 493 & & & & 0.78 & & & & 0.36 \\
\hline Mean T1:1553.0 & 93(18.9) & $2(16.7)$ & $24(15.9)$ & $67(20.3)$ & & $22(16.5)$ & $56(19.4)$ & $15(20.8)$ & \\
\hline Mean T2:1905.5 & 196(39.8) & $4(33.3)$ & $63(41.7)$ & $129(39.1)$ & & $49(36.8)$ & $113(39.2)$ & $34(47.2)$ & \\
\hline Mean T3: 2470.4 & 204(41.4) & $6(50.0)$ & $64(42.4)$ & $134(40.6)$ & & $62(46.6)$ & $119(41.3)$ & 23(31.9) & \\
\hline
\end{tabular}

*Significant at $\mathrm{p}<0.05$ for $\mathrm{x}^{2}$ tests.

${ }^{1} \mathrm{HH}$ : household, FS: food secure, FI: food insecure

Table 3. Eating behaviors based on the food and nutrition literacy scores in congnitive and skill domains

\begin{tabular}{|c|c|c|c|c|c|c|c|c|c|}
\hline & \multicolumn{9}{|c|}{ Food and nutrition literacy score } \\
\hline & \multicolumn{5}{|c|}{ Cognitive domain } & \multicolumn{3}{|c|}{ Skill domain } & \multirow[b]{2}{*}{$\mathrm{P}$ value* } \\
\hline & Total & low & moderate & high & $\mathrm{P}$ value* & low & moderate & high & \\
\hline & $\mathrm{N}(\%)$ & $\mathrm{N}(\%)$ & $\mathrm{N}(\%)$ & $\mathrm{N}(\%)$ & & $\mathrm{N}(\%)$ & $\mathrm{N}(\%)$ & $\mathrm{N}(\%)$ & \\
\hline Breakfast & 797 & & & & $0.009 *$ & & & & $<0.001 *$ \\
\hline every day & $475(59.6)$ & 13(61.9) & $115(50.2)$ & $347(63.4)$ & & $95(47.5)$ & 291(61.0) & $89(74.2)$ & \\
\hline 3-6 times a week & $188(23.5)$ & $4(19.0)$ & $66(28.8)$ & $118(21.6)$ & & $52(26.0)$ & $118(24.7)$ & $18(15.0)$ & \\
\hline 1-2 times a week & 95(11.9) & $2(9.5)$ & $39(17.0)$ & $54(9.9)$ & & $37(18.5)$ & $47(9.9)$ & $11(9.2)$ & \\
\hline never & $39(4.9)$ & $2(9.5)$ & $9(3.9)$ & $28(5.1)$ & & $16(8.0)$ & $21(4.4)$ & $2(1.7)$ & \\
\hline Lunch & 796 & & & & $<0.001 *$ & & & & $0.02 *$ \\
\hline every day & $635(79.8)$ & $17(81.0)$ & $158(69.0)$ & $460(84.2)$ & & $143(71.9)$ & $390(81.8)$ & $102(85.0)$ & \\
\hline 3-6 times a week & $110(13.8)$ & $2(9.5)$ & $53(23.1)$ & $55(10.1)$ & & $35(17.6)$ & $64(13.4)$ & $11(9.2)$ & \\
\hline 1-2 times a week & $47(5.9)$ & $2(9.5)$ & $16(7.0)$ & $29(5.3)$ & & $20(10.1)$ & $21(4.4)$ & $6(5.0)$ & \\
\hline never & $4(0.5)$ & $0(0)$ & $2(0.9)$ & $2(0.4)$ & & $1(0.5)$ & $2(0.4)$ & $1(0.8)$ & \\
\hline Dinner & 797 & & & & $0.04 *$ & & & & $0.002 *$ \\
\hline every day & $568(71.3)$ & $17(81.0)$ & $146(63.8)$ & $405(74.0)$ & & $127(63.5)$ & $351(73.6)$ & $90(75.0)$ & \\
\hline 3-6 times a week & 152(19) & $1(4.8)$ & $53(23.1)$ & $98(17.9)$ & & $38(19.0)$ & 94(19.7) & $20(16.7)$ & \\
\hline 1-2 times a week & $65(8.2)$ & $3(14.3)$ & $25(10.9)$ & $37(6.8)$ & & $29(14.5)$ & $28(5.9)$ & $8(6.7)$ & \\
\hline never & $12(1.5)$ & $0(0)$ & $5(2.2)$ & $7(1.3)$ & & $6(3.0)$ & $4(0.8)$ & $2(1.7)$ & \\
\hline Snack & 797 & & & & 0.83 & & & & 0.11 \\
\hline$\geq 3$ times a day & $273(34.3)$ & $8(38.1)$ & $76(33.2)$ & $189(34.6)$ & & $66(22.0)$ & $153(32.1)$ & $54(45.0)$ & \\
\hline 2 times a day & $225(28.2)$ & $6(28.6)$ & $62(27.1)$ & $157(28.7)$ & & $48(24.0)$ & $149(31.2)$ & $28(23.3)$ & \\
\hline 1 times a day & $202(25.3)$ & $4(19.0)$ & $66(28.8)$ & $132(24.1)$ & & $55(27.5)$ & $119(24.9)$ & $28(23.3)$ & \\
\hline never & $97(12.2)$ & $3(14.3)$ & $25(10.9)$ & $69(12.6)$ & & $31(15.5)$ & $56(11.7)$ & $10(8.3)$ & \\
\hline Water & & & & & 0.06 & & & & $0.01 *$ \\
\hline
\end{tabular}


Azam Doustmohammadian, et al: Food and Nutrition Literacy (FNLIT) and eating behavior

\begin{tabular}{|c|c|c|c|c|c|c|c|c|c|}
\hline \multirow{4}{*}{ Table 3 (continued). } & \multicolumn{8}{|c|}{ Food and nutrition literacy score } & \multirow[b]{3}{*}{ P value* } \\
\hline & \multicolumn{5}{|c|}{ Cognitive domain } & \multicolumn{3}{|c|}{ Skill domain } & \\
\hline & Total & low & moderate & high & $\mathrm{P}$ value* & low & moderate & high & \\
\hline & $\mathrm{N}(\%)$ & $\mathrm{N}(\%)$ & $\mathrm{N}(\%)$ & $\mathrm{N}(\%)$ & & $\mathrm{N}(\%)$ & $\mathrm{N}(\%)$ & $\mathrm{N}(\%)$ & \\
\hline$\geq 5$ cups a day & $581(72.9)$ & $17(81.0)$ & $157(68.9)$ & $407(74.3)$ & & $132(66.0)$ & $361(75.7)$ & $88(73.3)$ & \\
\hline 3- 4 cups a day & $149(18.7)$ & $3(14.3)$ & $47(20.6)$ & $99(18.1)$ & & $44(22.0)$ & $80(16.8)$ & $25(20.8)$ & \\
\hline 1- 2 cups a day & $59(7.4)$ & $0(0)$ & $19(8.3)$ & $40(7.3)$ & & $18(9.0)$ & $34(7.1)$ & $7(5.8)$ & \\
\hline 0 cup a day & $8(1)$ & $1(4.8)$ & $5(2.2)$ & $2(0.4)$ & & $6(3.0)$ & $2(0.4)$ & $0(0)$ & \\
\hline Tea/Coffee/Hot cacao & 798 & & & & 0.59 & & & & 0.52 \\
\hline$\geq 5$ times a week & 184(23) & $3(14.3)$ & $59(25.8)$ & $122(22.3)$ & & $43(21.5)$ & $119(24.9)$ & $22(18.3)$ & \\
\hline 3-4 times a week & $175(21.9)$ & $3(14.3)$ & $54(23.6)$ & $118(21.5)$ & & $44(22.0)$ & $105(22.0)$ & $26(21.7)$ & \\
\hline $1-2$ times a week & $306(38.3)$ & $11(52.4)$ & $82(35.8)$ & 213(38.9) & & $73(36.5)$ & $180(37.7)$ & $53(44.2)$ & \\
\hline never & $133(16.7)$ & $4(19.0)$ & $34(14.8)$ & $95(17.3)$ & & $40(20.0)$ & $74(15.5)$ & $19(15.8)$ & \\
\hline $\begin{array}{l}\text { Soft drinks/Industrial fruit } \\
\text { juices }\end{array}$ & 798 & & & & 0.46 & & & & 0.06 \\
\hline$\geq 5$ times a week & $61(7.6)$ & $3(14.3)$ & $20(8.7)$ & $38(6.9)$ & & $26(13.0)$ & $32(6.7)$ & $3(2.5)$ & \\
\hline 3-4 times a week & $122(15.3)$ & $2(9.5)$ & $37(16.2)$ & $83(15.1)$ & & $35(17.5)$ & $72(15.1)$ & $15(12.5)$ & \\
\hline 1-2 times a week & $329(41.2)$ & $11(52.4)$ & $98(42.8)$ & $220(40.1)$ & & $77(38.5)$ & $204(42.7)$ & $48(40.0)$ & \\
\hline never & $286(35.8)$ & $5(23.8)$ & $74(32.3)$ & 207(37.8) & & $62(31.0)$ & $170(35.6)$ & $54(45.0)$ & \\
\hline Sausage/Hamburger & 798 & & & & $<0.001^{*}$ & & & & $0.01 *$ \\
\hline$\geq 5$ times a week & $15(1.9)$ & $2(9.5)$ & $7(3.1)$ & $6(1.1)$ & & $7(3.5)$ & $8(1.7)$ & $0(0)$ & \\
\hline 3-4 times a week & $40(5)$ & $2(9.5)$ & $15(6.6)$ & $23(4.2)$ & & $11(5.5)$ & $27(5.6)$ & $2(1.7)$ & \\
\hline 1-2 times a week & $222(27.8)$ & $7(33.3)$ & $80(34.9)$ & $135(24.6)$ & & $68(34.0)$ & $120(25.1)$ & $34(28.3)$ & \\
\hline never & $522(65.3)$ & $10(47.6)$ & $127(55.5)$ & $384(70.1)$ & & $114(57.0)$ & $323(67.6)$ & $84(70.0)$ & \\
\hline Pizza & 798 & & & & 0.24 & & & & 0.14 \\
\hline$\geq 5$ times a week & $14(1.8)$ & $1(4.8)$ & $5(2.2)$ & $8(1.5)$ & & $6(3.0)$ & $8(1.7)$ & $0(0)$ & \\
\hline 3-4 times a week & $13(1.6)$ & $1(4.8)$ & $6(2.6)$ & $6(1.1)$ & & $6(3.0)$ & $6(1.3)$ & $1(0.8)$ & \\
\hline 1-2 times a week & $175(21.9)$ & $7(33.3)$ & $52(22.7)$ & $116(21.2)$ & & $50(25.0)$ & $98(20.5)$ & $27(22.5)$ & \\
\hline never & $596(74.7)$ & $12(57.1)$ & $166(72.5)$ & $418(76.3)$ & & $138(69.0)$ & $366(76.6)$ & $92(76.7)$ & \\
\hline French fries & 798 & & & & $<0.001^{*}$ & & & & $<0.001 *$ \\
\hline$\geq 5$ times a week & $49(6.1)$ & $6(28.6)$ & $23(10.0)$ & $20(3.6)$ & & $21(10.5)$ & $25(5.2)$ & $3(2.5)$ & \\
\hline 3-4 times a week & $101(12.7)$ & $2(9.5)$ & $33(14.4)$ & $66(12.0)$ & & $30(15.0)$ & $64(13.4)$ & $7(5.8)$ & \\
\hline 1-2 times a week & $389(48.7)$ & $6(28.6)$ & $109(47.6)$ & $274(50.0)$ & & $97(48.5)$ & $241(50.4)$ & $51(42.5)$ & \\
\hline never & $259(32.5)$ & $7(33.3)$ & 64(27.9) & $188(34.3)$ & & $52(26.0)$ & $148(31.0)$ & $59(49.2)$ & \\
\hline $\begin{array}{l}\text { Restaurant foods/ Fast } \\
\text { foods }\end{array}$ & 797 & & & & 0.12 & & & & 0.12 \\
\hline$\geq 5$ times a week & 13(1.6) & $0(0)$ & $9(4.0)$ & $4(0.8)$ & & $4(2.0)$ & $9(1.9)$ & $0(0)$ & \\
\hline 3-4 times a week & $37(4.6)$ & $1(4.8)$ & $10(4.4)$ & $26(4.8)$ & & $11(5.5)$ & $24(5.0)$ & $2(1.7)$ & \\
\hline 1-2 times a week & $236(29.2)$ & $8(38.1)$ & $69(30.1)$ & $159(29.1)$ & & $71(35.5)$ & $131(27.5)$ & $34(28.3)$ & \\
\hline never & $511(64.1)$ & $12(57.1)$ & $141(61.6)$ & $358(65.4)$ & & $114(57.0)$ & $313(65.6)$ & $84(70.0)$ & \\
\hline Sweet snacks & 798 & & & & $0.01 *$ & & & & $0.02 *$ \\
\hline$\geq 5$ times a week & $120(15)$ & $3(14.3)$ & $35(15.3)$ & $82(15.0)$ & & $31(1.5)$ & $76(15.9)$ & $13(10.8)$ & \\
\hline 3-4 times a week & $236(29.6)$ & $4(19.0)$ & $85(37.1)$ & $147(26.8)$ & & $67(33.5)$ & $142(29.7)$ & $27(22.5)$ & \\
\hline 1-2 times a week & $333(41.7)$ & $14(66.7)$ & $81(35.4)$ & $238(43.4)$ & & $74(37.0)$ & $206(43.1)$ & $53(44.2)$ & \\
\hline never & $109(13.7)$ & $0(0)$ & $28(12.2)$ & $81(14.8)$ & & $28(14.0)$ & $54(11.3)$ & $27(22.5)$ & \\
\hline Sugar & 798 & & & & 0.37 & & & & $0.03 *$ \\
\hline$\geq 5$ times a week & $193(24.2)$ & $6(28.6)$ & $68(29.7)$ & $119(21.7)$ & & $55(27.5)$ & $120(25.1)$ & $18(15.0)$ & \\
\hline 3-4 times a week & 191(23.9) & $5(23.8)$ & $52(22.7)$ & $134(24.5)$ & & $50(25.0)$ & $118(24.7)$ & $23(19.2)$ & \\
\hline 1-2 times a week & $284(35.6)$ & $6(28.6)$ & $77(33.6)$ & 201(36.7) & & $64(32.0)$ & $162(33.9)$ & $58(48.3)$ & \\
\hline never & $130(16.3)$ & $4(19.0))$ & $32(14.0)$ & $94(17.2)$ & & $31(15.5)$ & $78(16.3)$ & $21(17.5)$ & \\
\hline Honey/Jam & 798 & & & & $<0.001^{*}$ & & & & $<0.001 *$ \\
\hline$\geq 5$ times a week & $148(18.5)$ & $3(14.3)$ & $41(17.9)$ & $104(19.0)$ & & $27(13.5)$ & $88(118.4)$ & $33(27.5)$ & \\
\hline 3-4 times a week & $134(16.8)$ & $7(33.3)$ & $30(13.1)$ & $97(17.7)$ & & $29(14.5)$ & $74(15.5)$ & $31(25.8)$ & \\
\hline 1-2 times a week & $221(27.7)$ & $8(38.1)$ & $62(27.1)$ & $151(27.6)$ & & $49(24.5)$ & $145(30.3)$ & $27(22.5)$ & \\
\hline never & 295(37) & $3(14.3)$ & $96(41.9)$ & $196(35.8)$ & & $95(47.5)$ & $171(35.8)$ & $29(24.2)$ & \\
\hline Salty snacks & 798 & & & & 0.10 & & & & $<0.001 *$ \\
\hline$\geq 5$ times a week & $65(8.1)$ & $4(19.0)$ & $24(10.5)$ & $47(6.8)$ & & $26(13.0)$ & $33(6.9)$ & $6(5.0)$ & \\
\hline 3-4 times a week & $155(19.4)$ & $5(23.8)$ & $43(18.8)$ & $107(19.5)$ & & $39(19.5)$ & $98(20.5)$ & $18(15.0)$ & \\
\hline 1-2 times a week & $345(43.2)$ & $10(47.6)$ & $102(44.5)$ & $233(42.5)$ & & $96(48.0)$ & $210(43.9)$ & $18(15.0)$ & \\
\hline never & $233(29.2)$ & $2(9.5)$ & $60(26.2)$ & $171(31.2)$ & & $39(19.5)$ & $137(28.7)$ & $57(47.5)$ & \\
\hline
\end{tabular}

Notes: *Significant at $\mathrm{p}<0.05$ for $\mathrm{x}^{2}$ tests. 
Table 4. The adjusted ${ }^{\ddagger}$ odds ratios $(95 \% \mathrm{CI})$ of healthy eating behaviors ${ }^{\dagger}$ for FNLIT domains

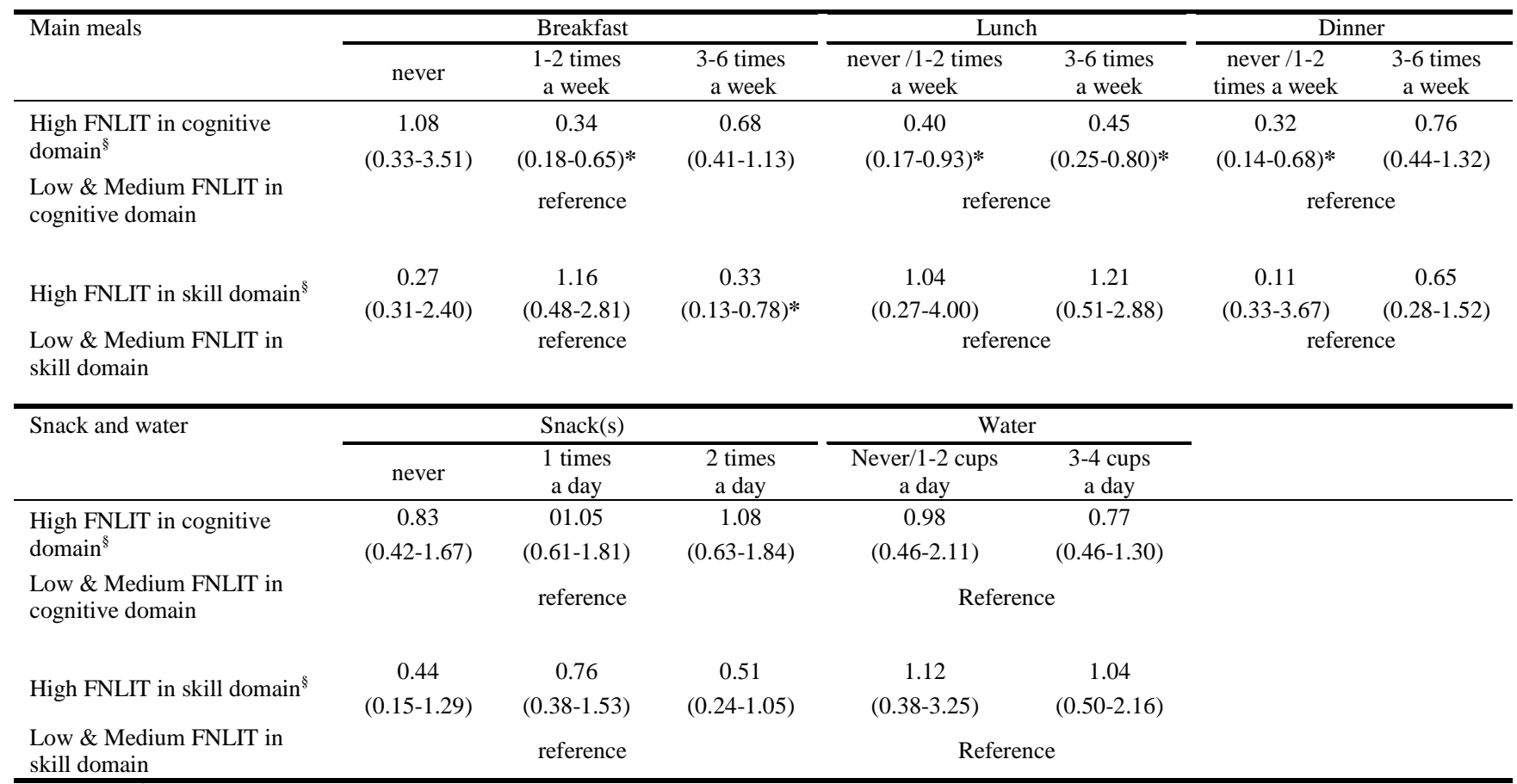

Notes: ${ }^{\dagger}$ Multinomial logistic model comparing eating behaviors category to $\geq 5$ cups group in water, to every day group in Breakfast, Lunch, dinner and $\geq \mathbf{3}$ times a day group in snack categories. FNLIT references category is moderate and low. ${ }^{\ddagger}$ Adjusted for sex, school status (governmental and nongovernmental), grade, birth rank, family size, ethnicity, parents age, parents' education, father job position, mother employment, Other income source of family members, weight status and calorie intake. *Significant at $\mathrm{p}<0.05$.

\section{Discussion}

Findings showed that high FNLIT scores in the cognitive and skill domains were associated to healthy eating behaviors. The findings were similar to those in previous studies, which showed that high food literacy/nutrition literacy was associated to frequencies of main meal consumption (30), preferences for healthy foods, decreased fast-food portion sizes and decreased consumption frequencies of packaged or processed snacks in school-age children and adolescents in developed and developing countries $(31,32)$. Children food preferences are established at younger ages and evolve around family cultural preferences, beliefs and attitudes (33) as well as their food environments and peer behaviors in schools (34). The FNLIT includes key roles in forming children preferences (35). Evidence have shown that involving children in healthy food preparation and improving their nutrition skills can lead to subsequent preferences for intake of healthy foods (36-38). Findings by Larson et al. revealed that higher levels of food and nutrition skills were associated to increased fruit and vegetable consumption and inversely linked to unhealthy food choices, including consumption of soft drinks and fried foods, in children and adolescents (39). Lack of food skills and confidence, specifically in cooking and food preparation (as FNLIT skill), seems as barriers to healthy eating behaviors (40, 41).
In the current study, unhealthy eating behaviors such as consumption sweet snacks and processed meats were quite prevalent between the children, similar to those of previous reports from Iran $(42,43)$ and other countries $(25,44)$. However, these were significantly lower in children with higher FNLIT scores. Despite the current government regulations, Iranian children are exposed to considerable numbers of food advertisements (45). Food producers highly affect most advertised foods and information they provide may not necessarily be interested by the publicity (46). In contrast, the easy access to junk foods in schools $(43,47,48)$ affects taste preferences of the children (49). Improving food and nutrition skills such as food advertising literacy, food purchasing behaviors and food label literacy can help children respond critically to such food media and make better food choices (50).

In the present study, some eating behaviors were linked to the cognitive or skill domain and some to both domains. Literacy is the concept beyond the pure knowledge that emphasizes functional aspects rather than knowledge (9). Nutrition knowledge may play fundamental but small roles in adopting healthy eating behaviors, while food skills are essential to translate knowledge into practice. Food and nutrition skills can be development if children include prior food and nutrition knowledges (51). However, development of skills typically provides knowledge as practicing them lead to acquisition of knowledge $(51,52)$. 
Table 5. The adjusted ${ }^{\ddagger}$ odds ratios $\left(95 \%\right.$ CI) of unhealthy eating behaviors ${ }^{\dagger}$ for FNLIT domains

\begin{tabular}{|c|c|c|c|c|c|c|c|c|c|c|c|c|}
\hline \multirow{2}{*}{ Sugary drinks } & \multicolumn{3}{|c|}{ Tea/Coffee/Hot cacao } & \multicolumn{6}{|c|}{ Soft drinks/ Industrial fruit juices } & \multicolumn{3}{|c|}{ Sausage/Hamburger } \\
\hline & never & $\begin{array}{c}1-2 \text { times } \\
\text { a week }\end{array}$ & $\begin{array}{c}\text { 3-4 times } \\
\text { a week }\end{array}$ & \multicolumn{2}{|c|}{ never } & $\begin{array}{r}1-2 \text { time } \\
\text { a week } \\
\end{array}$ & & \multicolumn{2}{|c|}{$\begin{array}{c}\text { 3-4 times } \\
\text { a week }\end{array}$} & \multicolumn{3}{|c|}{$\begin{array}{c}1-2 \text { times } \\
\text { a week }\end{array}$} \\
\hline High FNLIT in cognitive domain $^{\S}$ & $\begin{array}{c}1.30 \\
(0.65-2.60)\end{array}$ & $\begin{array}{c}0.91 \\
(0.53-1.57)\end{array}$ & $\begin{array}{c}0.76 \\
(0.42-1.40)\end{array}$ & \multicolumn{2}{|c|}{$\begin{array}{c}0.95 \\
(0.38-2.34)\end{array}$} & $\begin{array}{c}0.93 \\
(0.38-2.2\end{array}$ & & \multicolumn{2}{|c|}{$\begin{array}{c}0.98 \\
(0.37-2.58)\end{array}$} & \multicolumn{3}{|c|}{$(0.96-4.83) \quad$ reference $^{(0.47-2.52)}$} \\
\hline High FNLIT in skill domain ${ }^{\S}$ & $\begin{array}{c}0.78 \\
(0.27-2.23)\end{array}$ & $\begin{array}{c}1.70 \\
(0.81-3.57)\end{array}$ & $\begin{array}{c}1.34 \\
(0.57-3.15)\end{array}$ & $\begin{array}{r}7 \\
(0.90\end{array}$ & $\begin{array}{l}7 \\
3.03)\end{array}$ & $\begin{array}{c}4.50 \\
(0.54-37.3\end{array}$ & & $\begin{array}{r}5.16 \\
(0.58-45\end{array}$ & & \multirow{2}{*}{\multicolumn{3}{|c|}{ reference ${ }^{(0.53-12.81)}$}} \\
\hline Low \& Medium FNLIT in skill domain & & reference & & & & refe & ence & & & & & \\
\hline \multirow[t]{2}{*}{ Fast foods } & \multicolumn{3}{|c|}{ Pizza } & \multicolumn{6}{|c|}{ French fries } & \multicolumn{3}{|c|}{ Restaurant foods/ Fast foods } \\
\hline & Never & \multicolumn{2}{|c|}{$\begin{array}{l}\text { 1-2 times } \\
\text { a week }\end{array}$} & \multicolumn{2}{|c|}{ never } & \multicolumn{2}{|c|}{$\begin{array}{l}1-2 \text { times } \\
\text { a week }\end{array}$} & \multicolumn{2}{|c|}{$\begin{array}{l}\text { 3-4 times } \\
\text { a week }\end{array}$} & never & \multicolumn{2}{|c|}{$\begin{array}{l}\text { 1-2 times } \\
\text { a week }\end{array}$} \\
\hline High FNLIT in cognitive domain ${ }^{\S}$ & $\begin{array}{c}0.55 \\
(0.15-2.02)\end{array}$ & \multicolumn{2}{|c|}{$\begin{array}{c}0.42 \\
(0.11-1.63)\end{array}$} & \multicolumn{2}{|c|}{$\begin{array}{c}2.27 \\
(0.92-5.62)\end{array}$} & \multicolumn{2}{|c|}{$\begin{array}{c}1.89 \\
(0.80-4.46)\end{array}$} & \multicolumn{2}{|c|}{$\begin{array}{c}2.19 \\
(0.82-5.83)\end{array}$} & $\begin{array}{c}1.60 \\
(0.66-3.86)\end{array}$ & \multicolumn{2}{|c|}{$\begin{array}{c}1.22 \\
(0.49-3.01)\end{array}$} \\
\hline Low \& Medium FNLIT in cognitive domain & \multicolumn{3}{|c|}{ reference } & \multicolumn{6}{|c|}{ reference } & \multicolumn{3}{|c|}{ reference } \\
\hline High FNLIT in skill domain ${ }^{\S}$ & $\begin{array}{c}2.42 \\
(0.25-22.77)\end{array}$ & \multicolumn{2}{|c|}{$\begin{array}{c}2.28 \\
(0.23-23.06)\end{array}$} & \multicolumn{2}{|c|}{$\begin{array}{c}6.54 \\
(0.75-57.19\end{array}$} & \multicolumn{2}{|c|}{$\begin{array}{c}5.32 \\
(0.62-45.38)\end{array}$} & \multicolumn{2}{|c|}{$\begin{array}{c}1.38 \\
(0.12-15.68)\end{array}$} & $\begin{array}{c}6.07 \\
(0.72-51.439)\end{array}$ & $(0.4$ & -71 \\
\hline Low \& Medium FNLIT in skill domain & & reference & & & & refe & ence & & & & reference & \\
\hline Sweet \& Salty snacks & & Sweet snacks & & & Sugar & & & Honey/ Jam & & & Salty snacks & \\
\hline & never & $\begin{array}{l}1-2 \text { times } \\
\text { a week }\end{array}$ & $\begin{array}{l}\text { 3-4 times } \\
\text { a week }\end{array}$ & never & $\begin{array}{c}1-2 \text { times } \\
\text { a week }\end{array}$ & $\begin{array}{c}\text { 3-4 times } \\
\text { a week }\end{array}$ & never & $\begin{array}{c}1-2 \text { times } \\
\text { a week }\end{array}$ & $\begin{array}{l}\text { 3-4 times } \\
\text { a week }\end{array}$ & never & $\begin{array}{c}1-2 \text { times } \\
\text { a week }\end{array}$ & $\begin{array}{l}\text { 3-4 times } \\
\text { a week }\end{array}$ \\
\hline High FNLIT in cognitive domain $^{\S}$ & $\begin{array}{c}1.15 \\
(0.49-2.69)\end{array}$ & $\begin{array}{c}1.28 \\
(0.68-2.38)\end{array}$ & $\begin{array}{c}0.64 \\
(0.34-1.22)\end{array}$ & $\begin{array}{c}1.10 \\
(0.56-2.16)\end{array}$ & $\begin{array}{c}1.28 \\
(0.75-2.20)\end{array}$ & $\begin{array}{c}1.41 \\
(0.78-2.51)\end{array}$ & $\begin{array}{c}0.70 \\
(0.37-1.32)\end{array}$ & $\begin{array}{c}0.59 \\
(0.31-1.15)\end{array}$ & $\begin{array}{c}1.06 \\
0.50-2.26)\end{array}$ & $\begin{array}{c}2.16 \\
(0.95-4.94)\end{array}$ & $\begin{array}{c}1.83 \\
(0.85-3.93)\end{array}$ & $\begin{array}{c}2.58 \\
(1.09-6.13)^{*}\end{array}$ \\
\hline Low \& Medium FNLIT in cognitive domain & & reference & & & reference & & & reference & & & reference & \\
\hline High FNLIT in skill domain ${ }^{\S}$ & $\begin{array}{c}4.19 \\
(1.39-12.62)^{*}\end{array}$ & $\begin{array}{c}1.69 \\
(0.63-4.55)\end{array}$ & $\begin{array}{c}1.51 \\
(0.53-4.32)\end{array}$ & $\begin{array}{c}1.70 \\
0.63-4.52)\end{array}$ & $\begin{array}{c}2.25 \\
(1.02-4.96)\end{array}$ & $\begin{array}{c}1.02 \\
(0.41-2.55)\end{array}$ & $\begin{array}{c}0.46 \\
(0.20-1.01)\end{array}$ & $\begin{array}{c}0.43 \\
(0.18-1.03)\end{array}$ & $\begin{array}{c}1.14 \\
(0.51-2.59)\end{array}$ & $\begin{array}{c}4.27 \\
(0.89-20.30)\end{array}$ & $\begin{array}{c}1.64 \\
(0.34-7.86)\end{array}$ & $\begin{array}{c}2.35 \\
(0.46-11.94)\end{array}$ \\
\hline Low \& Medium FNLIT in skill domain & & reference & & & reference & & & reference & & & reference & \\
\hline
\end{tabular}

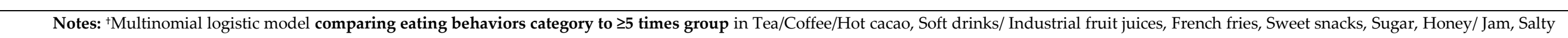

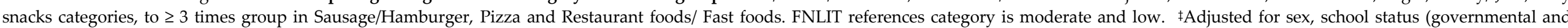

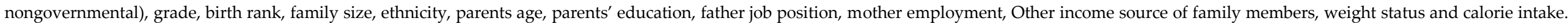

* Significant at $\mathrm{p}<0.05$. 
Links between the nutrition knowledge, skill and critical decision-making (which is conceptualized as food literacy) help children control their eating behaviors (9). In the current study, a considerable proportion of the students included low FNLIT scores in the skill domain, compared to the cognitive domain. This reveals that despite importance of the cognitive domain of FNLIT, effective strategies for the improvement of dietary skills are absent. This may be attributed to the fact that training in schools is based on the factual information and theoretical concepts. Content analysis of the Iranian primary school textbooks has shown that nutritional contents of the school textbooks are majorly theoretical rather than practical (53). These contents have led to students with high food and nutrition knowledges but with major gaps in performances and skills, resulting in unhealthy eating behaviors (54).

Based on the current evidence, individuals with higher self-efficacies are more likely to achieve desirable outcomes despite existed barriers (55). Building selfefficacy and sharing nutrition information with others through discussions and group activities in schools may be the most common strategies to improve children skills for better food choices (56). To improve student food skills, changing food ideas may best support children in making healthier food choices. Evidence show that school food atmosphere is a critical element in forming student dietary intakes and food choices (57). To the best of the authors' knowledge, no studies are available to investigate associations between FNLIT and eating behaviors of school-age children. Therefore, the current study includes significant values due to its novel findings and contents of numerous covariates to analyze FNLIT of eating behaviors. This obviously enables researchers to minimize the confounding effects of other factors. However, this study included limitations. The current study could not identify causal relationships such as possible reverse causalities due to its cross-sectional design. As the present study included frequencies of food intakes, quantities of the consumed foods could not be estimated. Future studies should include longitudinal approaches to investigate potentially causal contributions of FNLIT to children dietary intakes in large sample sizes. In contrast, statistically significant associations between dietary attributes and FNLIT were small in this study. Future studies in various social and cultural settings are necessary to investigate such associations.

\section{Conclusion}

The current study has added knowledge to the field by providing a common language for FNLIT. The present results are general reminders to schools of various learning needs of children. Furthermore, study highlights the necessity of continuous improvements in health education curriculum of schools in Iran, particularly in practical and skill-based lessons. Further studies with long-term followup plans are needed to better understand associations between FNLIT and eating behaviors.

\section{Ethics approval and consent to participate}

The study was approved by the National Nutrition and Food Technology Research Institute (NNFTRI) Ethics Committee (approval code: IR.SBMU.nnftri.Rec.1394.20). Written informed consents were signed by the students and their parents before the commencement of the survey.

\section{Authors' contributions}

$\mathrm{AD}$ was responsible for analyzing and interpreting data and drafting and editing the manuscript NO, NKM, HE-Z carried out the study design and analysis. AD, ZA, SE, collected data. AD, NO, NKM, MA1, MA2 and HE-Z participated in conceiving and designing the study, revising the manuscript and collecting data. All authors read and approved the final manuscript.

\section{Acknowledgement}

The authors express their appreciation to Shahid Beheshti University of Medical Sciences, National Nutrition and Food Technology Research Institute (NNFTRI), for funding the study. All coordinators and students participated in this study are appreciated.

\section{Financial disclosure}

The authors declared no financial interest.

\section{Funding/Support}

This work was supported by Shahid Beheshti University of Medical Sciences, National Nutrition and Food Technology Research Institute (NNFTRI) (grant no. 1394.20/16-102015).

\section{References}

1. Mendis S, Davis S, Norrving B. Organizational update: the world health organization global status report on noncommunicable diseases 2014; one more landmark step in the combat against stroke and vascular disease. Stroke. 2015;46(5):e121-2

2. Doustmohammadian A, Abdollahi M, Bondarianzadeh D, Houshiarrad A, Abtahi M. Parental determinants of overweight and obesity in Iranian adolescents: a national study. Iran J Pediatr. 2012;22(1):35-42.

3. Azadbakht L, Esmaillzadeh A. Macro and Micro-Nutrients Intake, Food Groups Consumption and Dietary Habits among Female Students in Isfahan University of Medical Sciences. Iran Red Crescent Med J. 2012;14(4):204-9.

4. Kelishadi R, Razaghi EM, Gouya MM, Ardalan G, Gheiratmand R, Delavari A, et al. Association of physical activity and the metabolic syndrome in children and adolescents: CASPIAN Study. Horm Res. 2007;67(1):46-52.

5. Kelishadi R, Ardalan G, Gheiratmand R, Majdzadeh R, Hosseini M, Gouya MM, et al. Thinness, overweight and 
obesity in a national sample of Iranian children and adolescents: CASPIAN Study. Child Care Health Dev. 2008;34(1):44-54.

6. Fung C, Kuhle S, Lu C, Purcell M, Schwartz M, Storey K, et al. From "best practice" to "next practice": the effectiveness of school-based health promotion in improving healthy eating and physical activity and preventing childhood obesity. Int J Behav Nutr Phys Act. 2012;9:27.

7. WHO. Jakarta Declaration on Health Promotion into the 21st Century. Switzerland, Geneva: World Health Organisation. 1997.

8. Zoellner J, Connell C, Bounds W, Crook L, Yadrick K. Nutrition literacy status and preferred nutrition communication channels among adults in the Lower Mississippi Delta. Prev Chronic Dis. 2009;6(4):A128.

9. Vidgen HA, Gallegos D. Defining food literacy and its components. Appetite. 2014;76:50-9

10. Velardo S. The Nuances of Health Literacy, Nutrition Literacy and Food Literacy. J Nutr Educ Behav. 2015;47(4):385-9 e1.

11. Laska MN, Larson NI, Neumark-Sztainer D, Story M. Does involvement in food preparation track from adolescence to young adulthood and is it associated with better dietary quality? Findings from a 10-year longitudinal study. Public Health Nutr. 2012;15(7):1150-8.

12. Zoellner J, You W, Connell C, Smith-Ray RL, Allen K, Tucker KL, et al. Health literacy is associated with healthy eating index scores and sugar-sweetened beverage intake: findings from the rural Lower Mississippi Delta. Journal of the American Dietetic Association. 2011;111(7):1012-20.

13. Oti JA. Food Literacy and Dietary Behaviour among Day Students of Senior High Schools in Winneba, Central Region of Ghana. Journal of Food and Nutrition Research. 2020;8(1):39-49.

14. Caraher M, Lloyd S, Lawton J, Singh G, Horsley K, Mussa F. A tale of two cities: A study of access to food, lessons for public health practice. Health Education Journal 2010;69(2):200-10.

15. Nutbeam D, Kickbusch I. Advancing health literacy: a global challenge for the 21st century. Health promotion international. 2000;15(3):183-4.

16. Doustmohammadian A, Omidvar N, Keshavarz Mohammadi N, Abdollahi M, Amini, M., Eini-Zinab H. Developing and validating a scale to measure Food and Nutrition Literacy (FNLIT) in elementary school children in Iran. PLoS One. 2017; (accepted)

17. Doustmohammadian A, Keshavarz Mohammadi N, Omidvar N, Amini M, Abdollahi M, Eini-Zinab H, et al. Food and nutrition literacy (FNLIT) and its predictors in primary schoolchildren in Iran. Health promotion international. 2018.

18. McGuire S. US Department of Agriculture and US Department of Health and Human Services, Dietary Guidelines for Americans, 2010. Washington, DC: US Government Printing Office, January 2011. Advances in Nutrition: An International Review Journal. 2011;2(3):293-4.

19. Rampersaud GC, Pereira MA, Girard BL, Adams J, Metzl JD. Breakfast habits, nutritional status, body weight and academic performance in children and adolescents. Journal of the American Dietetic Association. 2005;105(5):743-60
20. Vik FN, Bjornara HB, Overby NC, Lien $\mathrm{N}$ androutsos $\mathrm{O}$, Maes L, et al. Associations between eating meals, watching TV while eating meals and weight status among children, ages 10-12 years in eight European countries: the ENERGY cross-sectional study. Int J Behav Nutr Phys Act. 2013;10:58.

21. Croll JK, Neumark-Sztainer D, Story M. Healthy eating: what does it mean to adolescents? Journal of nutrition education. 2001;33(4):193-8.

22. Shi X, Tubb L, Fingers ST, Chen S, Caffrey JL. Associations of physical activity and dietary behaviors with children's health and academic problems. J Sch Health. 2013;83(1):1-7.

23. Geboers B, de Winter AF, Luten KA, Jansen CJ, Reijneveld SA. The association of health literacy with physical activity and nutritional behavior in older adults and its social cognitive mediators. Journal of health communication. 2014;19(sup2):61-76.

24. Bostock S, Steptoe A. Association between low functional health literacy and mortality in older adults: longitudinal cohort study. BMJ. 2012;344:e1602.

25. Kim SY, Sim S, Park B, Kong IG, Kim JH, Choi HG. Dietary Habits Are Associated With School Performance in Adolescents. Medicine (Baltimore). 2016;95(12):e3096.

26. Aadahl M, Jørgensen T. Validation of a new self-report instrument for measuring physical activity. Medicine and science in sports and exercise. 2003;35(7):1196-202.

27. Kelishadi R, Rabiei K, Khosravi A, Famouri F, Sadeghi M, Rouhafza $\mathrm{H}$, et al. Assessment of physical activity of adolescents in Isfahan. 2001.

28. Bickel G, Nord M, Price C, Hamilton W, Cook J. Guide to measuring household food security. Guide to Measuring Household Food Security in the United States: Revised 2000 No 6 March 2000 USDA, Food and Nutrition Services, Alexandria, VA. 2000.

29. Rafiei M, Nord M, Sadeghizadeh A, Entezari MH. Assessing the internal validity of a household survey-based food security measure adapted for use in Iran. Nutrition journal. 2009;8(1):1.

30. Utter J, Denny S, Lucassen M, Dyson B. Adolescent Cooking Abilities and Behaviors: Associations With Nutrition and Emotional Well-Being. J Nutr Educ Behav. 2016;48(1):35-41 e1.

31. Robson SM, Stough CO, Stark LJ. The impact of a pilot cooking intervention for parent-child dyads on the consumption of foods prepared away from home. Appetite. 2016;99:177-84.

32. Hersch D, Perdue L, Ambroz T, Boucher JL. The impact of cooking classes on food-related preferences, attitudes and behaviors of school-aged children: a systematic review of the evidence, 2003-2014. Prev Chronic Dis. 2014;11:E193.

33. Savage JS, Fisher JO, Birch LL. Parental influence on eating behavior: conception to adolescence. The Journal of Law, Medicine \& Ethics. 2007;35(1):22-34.

34. Ronto R, Ball L, Pendergast D, Harris ND. Food literacy at secondary schools in Australia. Journal of School Health. 2016;86(11):823-31.

35. Ballance D, Webb N. For the mouths of babes: nutrition literacy outreach to a child care center. Journal of consumer health on the Internet. 2015;19(1):1-12.

36. Birch LL, Doub AE. Learning to eat: birth to age 2 y. The American journal of clinical nutrition. 2014;99(3):723S-8S. 
37. Korinek EV, Bartholomew JB, Jowers EM, Latimer LA. Fruit and vegetable exposure in children is linked to the selection of a wider variety of healthy foods at school. Maternal \& child nutrition. 2015;11(4):999-1010.

38. Cooke L. The importance of exposure for healthy eating in childhood: a review. . Journal of Human Nutrition \& Dietetics. 2007;20(4):294-301.

39. Larson NI, Story M, Eisenberg ME, Neumark-Sztainer D. Food preparation and purchasing roles among adolescents: associations with sociodemographic characteristics and diet quality. J Am Diet Assoc. 2006;106(2):211-8.

40. Fulkerson JA, Kubik MY, Rydell S, Boutelle KN, Garwick A, Story M, et al. Focus groups with working parents of schoolaged children: what's needed to improve family meals? J Nutr Educ Behav. 2011;43(3):189-93.

41. Nicklas TA, Jahns L, Bogle ML, Chester DN, Giovanni M, Klurfeld DM, et al. Barriers and facilitators for consumer adherence to the dietary guidelines for Americans: the HEALTH study. J Acad Nutr Diet. 2013;113(10):1317-31.

42. Amini M, Dadkhah-Piraghaj M, Abtahi M, Abdollahi M, Houshiarrad A, Kimiagar M. Nutritional assessment for primary school children in tehran: an evaluation of dietary pattern with emphasis on snacks and meals consumption. Int $\mathbf{J}$ Prev Med. 2014;5(5):611-6.

43. Kelishadi R, Ardalan G, Gheiratmand R, Gouya MM, Razaghi EM, Delavari A, et al. Association of physical activity and dietary behaviours in relation to the body mass index in a national sample of Iranian children and adolescents: CASPIAN Study. Bull World Health Organ. 2007;85(1):1926.

44. Braithwaite I, Stewart AW, Hancox RJ, Beasley R, Murphy R, Mitchell EA. Fast-food consumption and body mass index in children and adolescents: an international cross-sectional study. BMJ Open. 2014;4(12):e005813.

45. Amini M, Mohsenian-Rad M, Kimiagar M, Ghaffarpour M, Omidvar N, Mehrabi Y. Food advertising on Iranian children's television: a content analysis and an experimental study with junior high school students keywords. Ecology of food and nutrition. 2005;44(2):123-33.

46. Harris JL, Pomeranz JL, Lobstein T, Brownell KD. A crisis in the marketplace: how food marketing contributes to childhood obesity and what can be done. Annu Rev Public Health. 2009;30:211-25
47. Kolahdooz F SR, Naghavi M, Abdollahi Z. Junk food consumption: An indicator of changing dietary habit in Iranian children. Asia Pac J Clin Nutr. 2004;13(Suppl):S121.

48. Foroughzadeh S. A study of public schools students consumption of school buffet snacks. Journal of Research and Health. 2013;3(2):388-94.

49. Boyland EJ, Halford JC. Television advertising and branding. Effects on eating behaviour and food preferences in children. Appetite. 2013;62:236-41.

50. Liao LL, Lai IJ, Chang LC, Lee CK. Effects of a food advertising literacy intervention on Taiwanese children's food purchasing behaviors. Health Educ Res. 2016;31(4):509-20.

51. Worsley A. Nutrition knowledge and food consumption: can nutrition knowledge change food behaviour? Asia Pac J Clin Nutr. 2002;11 Suppl 3:S579-85.

52. Ronto R, Ball L, Pendergast D, Harris N. Adolescents perspectives on food literacy and its impact on their dietary behaviours. Appetite. 2016;107:549-57.

53. Omidvar N, Amini M, Zahedi-Rad M, Khatam A. Content analysis of school textbooks in a view of improving nutritional knowledge and skills of primary school children. The 2th international and the 14th Iranian nutrition congress. 4-7 September,Tehran, Iran. 2016.

54. FAO. 2020. School-based food and nutrition education - A white paper on the current state, principles, challenges and recommendations for low- and middle-income countries. Rome. https://doi.org/10.4060/cb2064en.

55. Linde JA, Rothman AJ, Baldwin AS, Jeffery RW. The impact of self-efficacy on behavior change and weight change among overweight participants in a weight loss trial. Health Psychology. 2006;25(3):282.

56. Cha E, Kim KH, Lerner HM, Dawkins CR, Bello MK, Umpierrez $\mathrm{G}$, et al. Health literacy, self-efficacy, food label use and diet in young adults. Am $J$ Health Behav. 2014;38(3):331-9.

57. Ronto R, Ball L, Pendergast D, Harris N. Environmental factors of food literacy in Australian high schools: views of home economics teachers. International Journal of Consumer Studies. 2017;41(1):19-27.

58. Sumner, J. (ed.). Learning, Food and Sustainability: Sites for Resistance and Change, Palgrave Macmillan, New York, 2016. 